\title{
Simultaneous measurements of thermal diffusivity, thermal conductivity and thermopower with application to copper and ceramic superconductors
}

\author{
H. Bougrine ${ }^{1,2}$, J.F. Geys ${ }^{1, a}$, S. Dorbolo ${ }^{2}$, R. Cloots ${ }^{3}$, J. Mucha ${ }^{4}$, I. Nedkov ${ }^{5}$, and M. Ausloos ${ }^{2, b}$ \\ 1 S.U.P.R.A.S., Montefiore Electricity Institute, B28, University of Liège, 4000 Liège, Belgium \\ 2 S.U.P.R.A.S., Physics Institute, B5, University of Liège, 4000 Liège, Belgium \\ 3 S.U.P.R.A.S., Chemistry Institute, B6, University of Liège 4000 Liège, Belgium \\ 4 Institute for Structural and Low Temperature Research, Polish Academy of Sciences, 50950 Wroclaw, Poland \\ ${ }^{5}$ Institute of Electronics, Bulgarian Academy of Sciences, 72, Tsarigradsko Chaussee, 1784 Sofia, Bulgaria
}

Received 9 November 1998 and Received in final form 12 July 1999

\begin{abstract}
A home made experimental set-up allows us to measure the thermal conductivity, the thermopower and the thermal diffusivity simultaneously in the temperature range $(20-300 \mathrm{~K})$. Therefore the specific heat can be deduced. The role of a radiation shield is shown to be relevant. Tests of the system are made on a $99.9 \%$ pure $\mathrm{Cu}$ sample and two polycrystalline cuprate ceramics for illustration. Without any complicated optimisation, the technique indicates much promise already due to its efficiency and rapidity.
\end{abstract}

PACS. 06.30.-k Measurements common to several branches of physics and astronomy -

72.15.Jf Thermoelectric and thermomagnetic effects

\section{Introduction}

To measure the electrical resistivity of metallic and semiconducting compounds is a well-known material test procedure. Phenomena occurring under thermal gradient conditions are less popular because much more difficult to describe and to interpret. One reason is the difficulty to obtain reliable data on the same sample under the same experimental conditions. Therefore to measure the thermal conductivity and the thermoelectric power simultaneously has been a great challenge $[1,2]$. Another interesting and fundamental property is the specific heat which is a static (equilibrium) property. Its importance in physics is well-known because it indicates the presence or not of phase transitions from a thermodynamic point of view. Related to these properties is the thermal diffusivity coefficient which is a hybrid property. Not much is known on that quantity from a theoretical point of view. It might be that this is due to the lack of precision of the relevant data.

Our endeavour has been to measure simultaneously three properties, i.e. the thermal diffusivity together with the thermoelectric power and thermal conductivity which for the latter two has been already demonstrated to be

\footnotetext{
a Present address: Techspace-Aero, Department of Physics, 121 Route de Liers, 4041 Milmort, Belgium

b e-mail: ausloos@gw.unipc.ulg.ac.be
}

feasible [1]. This simultaneity should be an advantage because of the rapidity of execution, the smallest number of sample handlings, and the certainty about identical physical conditions during measurements. A major test is next to derive the specific heat from the combination of data.

We will show that we can implement the Kennedy [3] transient heat flow method, reducing at most the radiation losses in order to derive quite good data on the thermal diffusivity simultaneously measured with the thermal conductivity and the thermoelectric power. For a first comparison with other works and error estimates we have measured the thermal diffusivity of an ordinary $\mathrm{Cu}$ wire. Notice that to our knowledge there is no direct experimental thermal diffusivity data of copper at low temperature $(T<100 \mathrm{~K}),-$ except through the ratio of independent measurements of the thermal conductivity and the specific heat.

We have then tested whether the technique can be used on two polycrystalline superconducting ceramics in order to reveal minute features. The results are quite satisfactory and it can be hoped that they can thus be applied to other or better samples.

In Section 2 we present and discuss the experimental considerations. In Section 3 we report the experimental results. They are discussed in Section 4. A brief conclusion is found in Section 5 . 


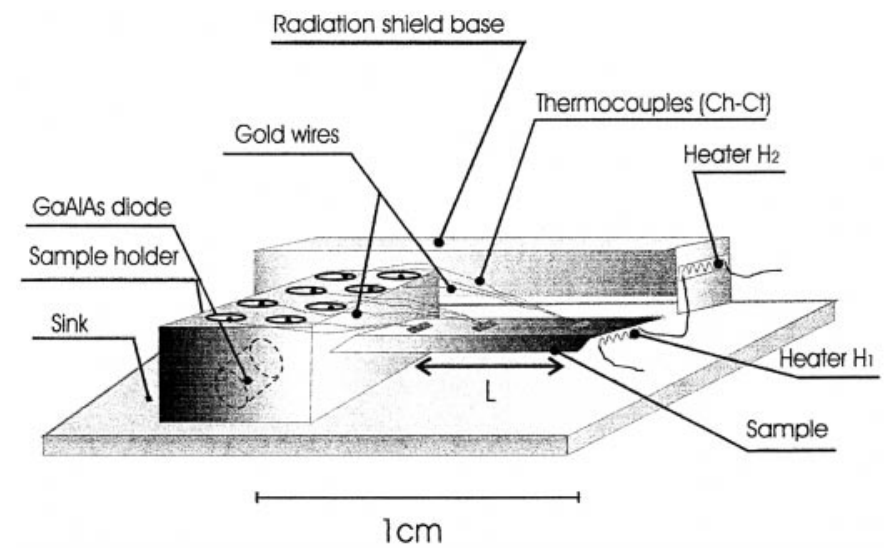

Fig. 1. Experimental set-up to measure simultaneously the thermal diffusivity, thermal conductivity and thermopower at low temperature. The sample, sample holder, and sink are entirely covered by the radiation shields.

\section{Experimental considerations}

\subsection{Set-up}

A schematic view of the experimental chamber set-up and sample holder which are used for measuring the thermal conductivity $\kappa(T)$, the thermopower $S(T)$ and the thermal diffusivity $\alpha(T)$ is shown in Figure 1 . The set-up is derived from that allowing the simultaneous measurements of $\kappa(T)$ and $S(T)$ as in reference [2].

The heater $\left(\mathrm{H}_{1}\right)$ is made of thin constantan film resistance $(150 \Omega)$, the latter value being measured by the four-probe method. The film is glued by GE7031 varnish on one face of the sample such that the sample heater $\left(\mathrm{H}_{1}\right)$ supplies a uniform heat flow through the sample. The other extremity of the sample is glued to the sample holder by means of cigarette paper and GE7031 varnish in order to reduce the thermal resistance between the sample and the sample holder. Indeed the thermal conductivity of the GE7031 is $0.1 \mathrm{~W} / \mathrm{m} . \mathrm{K}$ between 1 and $300 \mathrm{~K}$ [4]. Four phosphorus bronze wires (length $=10 \mathrm{~cm}, \phi=50 \mu \mathrm{m}$, $R=10 \Omega$ ) are used for carrying the current and measuring the voltage drop on the sample heater $\left(\mathrm{H}_{1}\right)$. The temperature at (three) different positions on the sample is measured through absolute chromel- constantan (Ch-Ct) calibrated thermocouples (length $=15 \mathrm{~cm}, \phi=50 \mu \mathrm{m}$ ) which are thermally anchored on the sink and the sample holder (wound and glued around them). We have included a copper radiation shield covering the sample. The shield temperature is controlled by a constantan $(150 \Omega)$ film resistance $\left(\mathrm{H}_{2}\right)$, and is maintained at nearly the sample and sample holder temperature. In order to reduce the conduction thermal losses the heater $\mathrm{H}_{1}$ and $\mathrm{H}_{2}$ are connected through the phosphorus bronzes wires themselves.

In this report the thermal diffusivity measurement requires a current pulse (about one second) when raising the sample temperature, such that it is difficult to keep the sample and the radiation shield at the same temperature in view of the relaxation times of both systems. Moreover the mass of the radiation shield is chosen to be three times bigger than the mass of the examined sample. Thus the temperature increase of the radiation shield cannot exceed the temperature of the sample. For the thermal conductivity measurement, we adopt the same technique with a $5 \mathrm{~mA}$ current (applied during about ten minutes) as required to reach a steady state. In order to reduce the radiation thermal losses and allow for a faster temperature equilibration as much as possible the current injected into the sample is the same as that flowing through the radiation shield heater (see Fig. 1). In our case the radiation thermal losses are thus reduced because of the permanently controlled temperature on the radiation shield and sample holder.

A close He gas circuit allows the system to be cooled down from room temperature to $20 \mathrm{~K}$, but we study samples mainly between 40 and $220 \mathrm{~K}$ where most of the interesting physical features appear. The whole experimental chamber is evacuated to $10^{-8}$ mbar by a turbo-molecular pump.

\subsection{Measurement method}

The technique described in this report utilizes and adapts the Kennedy transient heat flow method [3] for the thermal diffusivity. In this method, a sudden temperature change is made at one end of the sample. The details of the method have already been discussed elsewhere $[10,11]$. The thermal conductivity and the thermopower are measured using the steady-state [5] and differential [6] method respectively. Under these experimental conditions, the thermal diffusivity $\alpha(T)$, thermal conductivity $\kappa(T)$ and thermopower $S(T)$ of any solid sample can be measured simultaneously.

Consider non steady-state conditions of a unidirectional heat flow in an isotropic medium. The differential equation that gives the temperature rise at any position $x$ and time $t$ for a sample supposed to have a uniform cross section is

$$
\alpha \frac{\partial^{2} \theta}{\partial x^{2}}=\frac{\partial \theta}{\partial t}+\mu \theta
$$

where $\alpha=\kappa / d c_{\mathrm{p}}$, is the thermal diffusivity, and $\kappa$ the thermal conductivity, $d$ the density, $c_{\mathrm{p}}$ the specific heat at constant pressure, $\theta$ the change in temperature and $\mu$ is the coefficient of surface heat loss which takes into account any heat loss by radiation, conduction and convection. Moreover the heat loss has been considered to be proportional to $\theta$ but this is so without any great loss of generality, as long as the $x$ and $t$ variations are small. Moreover in the set-up described above the thermal losses have been reduced experimentally as much as possible, i.e. due to the radiation shield and controlled feedback of the temperature differences. Therefore the coefficient $\mu$ can be neglected in equation (1), since (i) the radiation shield is kept at the same temperature of the sample, (ii) the wires are long, thin and thermally anchored, and (iii) the vacuum is rather high.

The Crank-Nicolson implicit method [12], and a high speed computer are then used to solve numerically 
equation (1). For simplicity the $x$-axis and the $t$-axis are divided into equal intervals $\Delta x$ and $\Delta t$ respectively (the so-called finite difference method). If we let $\theta_{i, j}$ to be an approximation to $\theta\left(t_{i}, x_{j}\right)$, where $t_{i}=i \Delta t$ and $x_{j}=j \Delta x$. A numerical difference approximation to equation (1) can therefore be written

$$
\begin{aligned}
& 2(1+\beta) \theta_{i+1, j}-\beta\left(\theta_{i+1, j-1}+\theta_{i+1, j+1}\right)= \\
& 2(1-\beta) \theta_{i, j}+\beta\left(\theta_{i, j-1}+\theta_{i, j+1}\right)
\end{aligned}
$$

where $\beta=\alpha \Delta t /(\Delta x)^{2}$ is a kind of normalized thermal diffusivity. In our set-up three thermocouples are attached to the sample at $x=0, x=L / 2$ and $x=L$, (Fig. 1 ). The temperature of the three absolute thermocouples is denoted $\operatorname{th}_{1}(t), \operatorname{th}_{2}(t)$ and $\operatorname{th}_{3}(t)$ respectively at $x=0$, $x=L / 2$ and $x=L$. They can be represented as follows

$$
\begin{aligned}
\theta_{0, j} & =0 \\
\theta_{i, 0} & =\operatorname{th}_{1}(i) \\
\theta_{i, L} & =\operatorname{th}_{3}(i) .
\end{aligned}
$$

The position of the second thermocouple does not affect much the precision of the results. The temperature at the outer thermocouples at $x=0$ and $x=L$ determines the boundary conditions for the heat-flow equation (2) which can be solved then for the midpoint temperature (at $x=L / 2$ ) by assuming various and realistic values of the thermal diffusivity. Interpolation and comparison are made for each calculated temperature with the observed temperature at $x=L / 2$. The value of $\alpha$ which gives the smallest difference between the calculated and the observed temperature is accepted to be the thermal diffusivity of the sample. Thus by taking into account the boundary conditions described above, we can calculate all the temperature changes $\theta\left(t_{i}, x_{j}\right)$ situated between the extremities of the sample at $x=0$ and $x=L$.

The thermal run goes as follows: for the thermal diffusivity we stabilize the temperature of the whole experimental chamber through a temperature controller. After measuring during a short time (2 second) the residual potential which corresponds to some residual temperature difference on the thermocouple wires. Without taking into account the residual potential which depends on the temperature we can expect an error bar of the order of $15 \%$ as discussed in reference [1]. A one second heat pulse is next applied and the temperature is measured as a function of time during $100 \mathrm{~s}$ or more depending on the thermal properties of the sample. We emphasize here that the temperature data as a function of time is taken successively at each thermocouple in order to take a precise and optimum number of points. After the heat pulse is applied, the thermal diffusivity is deduced from a measurement of the change in temperature at each thermocouple as a function of time. We report here below the best values of $\alpha$ after having made several trials. Those "best values" are certainly specific to the set-up but are likely to be good orders of magnitude for similar set-ups. The best results have been obtained for a $375 \mathrm{~mW}$ heat pulse and a $1 \mathrm{~s}$ current pulse. Such values of the heat pulse and the time current pulse are large enough to raise the mean temperature of the sample by one degree and small enough to keep the sample in the transient heat flow regime. The amplitude and the duration of the heat pulse have been found to be an increasing function of the density of the sample. The discretisation of the time division is $\Delta t=0.25 \mathrm{~s}$ and the space division is $\Delta x=0.25 \mathrm{~mm}$.

For measuring the thermal conductivity and the thermopower the stability of the temperature is rechecked at each thermocouple perturbed by the previous heat pulse. The detected changes in temperature have been found to be about $1 \%$. After measuring again the residual potential and after the steady state is considered to be wellestablished, (after about 10 minutes or more depending of sample) a new temperature gradient between the first thermocouple $(x=0)$ and the second thermocouple $(x=L / 2)$ is applied.

We point out here that the numerical solution of the thermal diffusivity needs a long time (15 minutes). By the way, we use this time interval for guaranteeing the stabilization of the whole experimental chamber through increasing or decreasing the temperature of the sample holder.

\subsection{Experimental error estimates}

In order not to increase the thermal losses we emphasize that we use a small heat pulse amplitude on the sample. The relative error on the thermal conductivity $\kappa$ is $\Delta \kappa / \kappa=\Delta P_{\mathrm{m}} / P+\Delta P_{\mathrm{L}} / L+\Delta(\Delta T) / \Delta T+\Delta L / L+\Delta A / A$, where $P_{\mathrm{m}}$ is the applied power, $P_{\mathrm{L}}$ are the thermal losses, $\Delta T$ is the temperature gradient, $L$ is the distance between thermocouples, and $A$ is the cross section area of the sample. The relative error size is estimated to be $\approx 8 \%$ resulting from estimates i.e. $2 \%, 1 \%, 1 \%, 3 \%$ and $0.3 \%$ for the applied power, the thermal losses, the temperature gradient, the distance between thermocouples, and the cross section area of the sample respectively considering the same arguments as those found in reference [1]. Due to the choice of the size (section and length) of the thermocouple wires the conduction heat losses are negligible. For the thermal diffusivity no precise value of the absolute quantity of heat introduced into the sample is even necessary. Thus the error can be estimated to be $2 \%$ less than that for the thermal conductivity. Finally the estimated error for measuring the thermopower from $S=S_{\mathrm{Au}}+(\Delta V / \Delta T)$ is estimated to be $\approx 2 \%$, i.e. the error is $1 \%$, and $1 \%$ respectively for the gold wire thermopower $S_{\mathrm{Au}}$ and the temperature gradient $\Delta T$.

\section{Experimental results}

To test the set-up and validate our technique we have used three samples in this study, (i) a pure copper wire (length $=10 \mathrm{~mm}, \phi=0.4 \mathrm{~mm}$ ) and two ceramic superconductors $\left(10 \times 2 \times 2 \mathrm{~mm}^{3}\right)$, i.e. (ii) $\mathrm{Y}_{1-x} \mathrm{Ca}_{x} \mathrm{Ba}_{2} \mathrm{Cu}_{3} \mathrm{O}_{7-\delta}$ $(x=0.1)$ and (iii) $\mathrm{Dy}_{1} \mathrm{Ba}_{2} \mathrm{Cu}_{3} \mathrm{O}_{7-\delta}$. The latter two systems are called YCaBCO and DyBCO for brievity in the figures. The copper wire has $99.9 \%$ purity and was obtained from Goodfellow Corp. The $\mathrm{Y}_{1-x} \mathrm{Ca}_{x} \mathrm{Ba}_{2} \mathrm{Cu}_{3} \mathrm{O}_{7-\delta}$ 


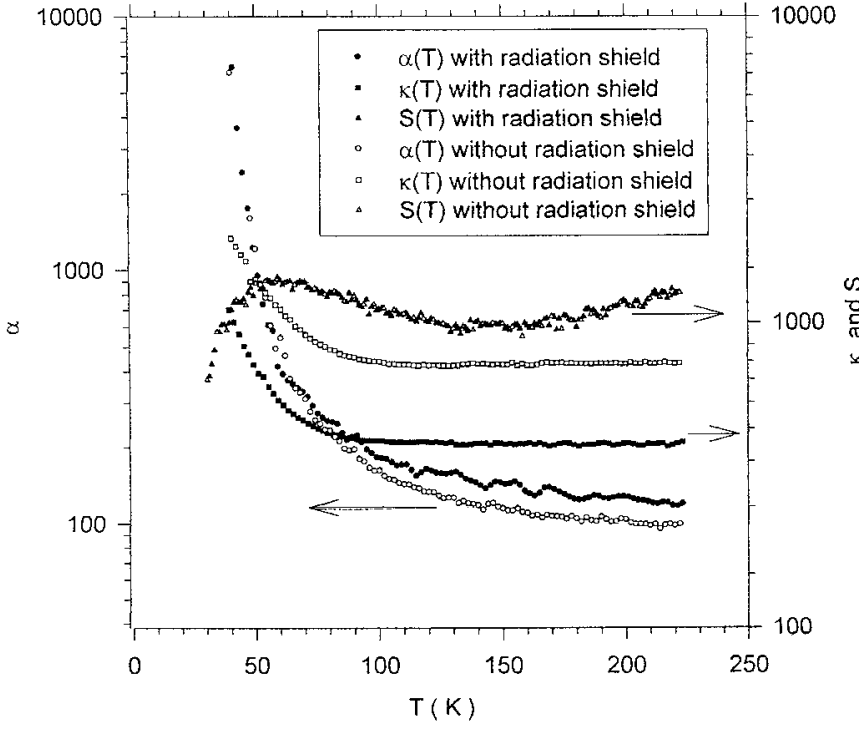

Fig. 2. Comparison between the thermal diffusivity $\alpha(T)$ and the thermal conductivity $\kappa(T)$ of a $99.9 \%$ pure $\mathrm{Cu}$ for measurements taken in presence or not of the radiation shield.

$(x=0.1)$ was prepared in S.U.P.R.A.S group [7] by the conventional solid state reaction method. Stoichiometric amounts of $\mathrm{Y}_{2} \mathrm{O}_{3}, \mathrm{CaCO}_{3}, \mathrm{BaCO}_{3}$ and $\mathrm{CuO}$ were mixed together and heat treated at $930{ }^{\circ} \mathrm{C}$ for 48 hours with two intermediary grinding. The resulting powder was then pressed in a pellet shape and annealed in an oxygen atmosphere from 950 to $450{ }^{\circ} \mathrm{C}$ during 36 hours. Xray diffraction analysis shows that the sample is nearly monophasic, containing some extra $\mathrm{BaCuO}_{2}$ phase. The $\mathrm{Dy}_{1} \mathrm{Ba}_{2} \mathrm{Cu}_{3} \mathrm{O}_{7-\delta}$ was prepared using $\mathrm{Y}_{2} \mathrm{O}_{3}, \mathrm{Dy}_{2} \mathrm{O}_{3}, \mathrm{CuO}$, $\mathrm{BaCO}_{3}[8,9]$. Stoichiometric amounts of initial materials were homogenized by wet milling. The wet milling was made in a Fritsh ball-mill with ethanol medium for four hours. Prepared powders were dried and then milled in KM vibration mill for $30 \mathrm{~min}$. The powders were pressed into $\phi=15 \mathrm{~mm}$ pellet under $5 \mathrm{MPa}$ pressure. The pellet was sintered at $950{ }^{\circ} \mathrm{C}$ with isothermal delay of 24 hours. A vibration mill was used for the second milling and the grain size was controlled. We used powder with typical linear grain size $c a .0 .001 \mathrm{~mm}$ for the last pressing. The powders were pressed into $\phi=10 \mathrm{~mm}$ pellet under $3.5 \mathrm{MPa}$ pressure. Each pellet was sintered at $950{ }^{\circ} \mathrm{C}$ during 120 hours. It was cooled down to $650{ }^{\circ} \mathrm{C}$ with a isothermal delay of 24 hours. Then the pellet was extracted from the oven and let to cool down to room temperature.

Figure 2 allows us a comparison of the experimental points of the thermal diffusivity $\alpha(T)$, thermal conductivity $\kappa(T)$, and the thermopower $S(T)$ for a $99.9 \%$ pure Goodfellow $\mathrm{Cu}$ sample as a function of temperature in presence or in absence of the radiation shield (Fig. 2). The specific heat as determined from the expression $\alpha=\kappa / d c_{\mathrm{p}}$ where $d=8.9 \mathrm{~g} / \mathrm{cm}^{3}$ is the density of copper [13], is shown in Figure 3 for both experimental conditions. An inset shows $c_{\mathrm{p}} / T$ vs. $T^{2}$. Following the best conditions as described above, we also compare the thermal conductivity and thermopower of two polycrystalline

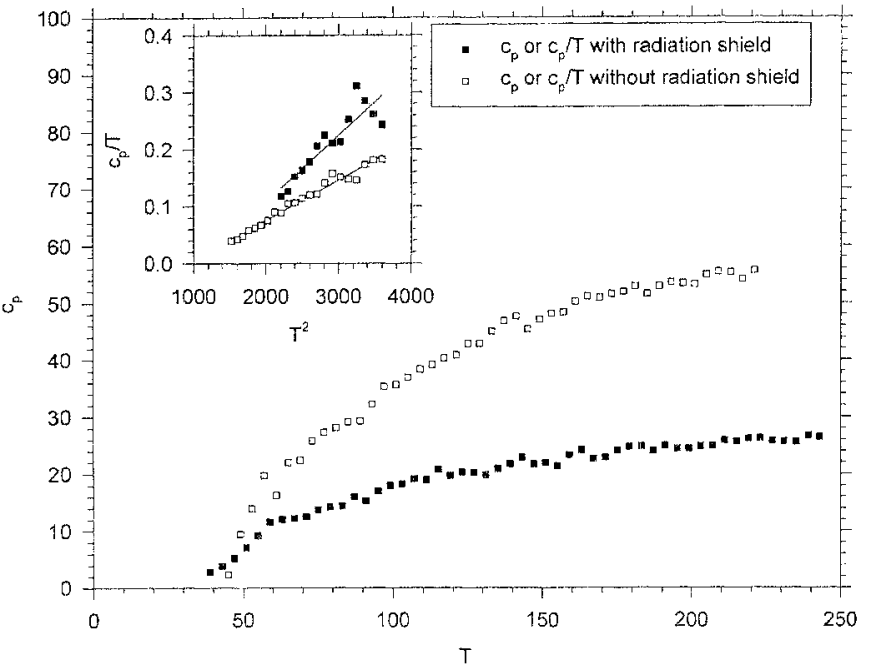

Fig. 3. Comparison between specific heat $c_{\mathrm{p}}(T)$ data of a 99.9\% pure $\mathrm{Cu}$ for measurements taken in presence or not of the radiation shield. Inset: comparisons on a $c_{\mathrm{p}} / T$ vs. $T^{2}$ plot.

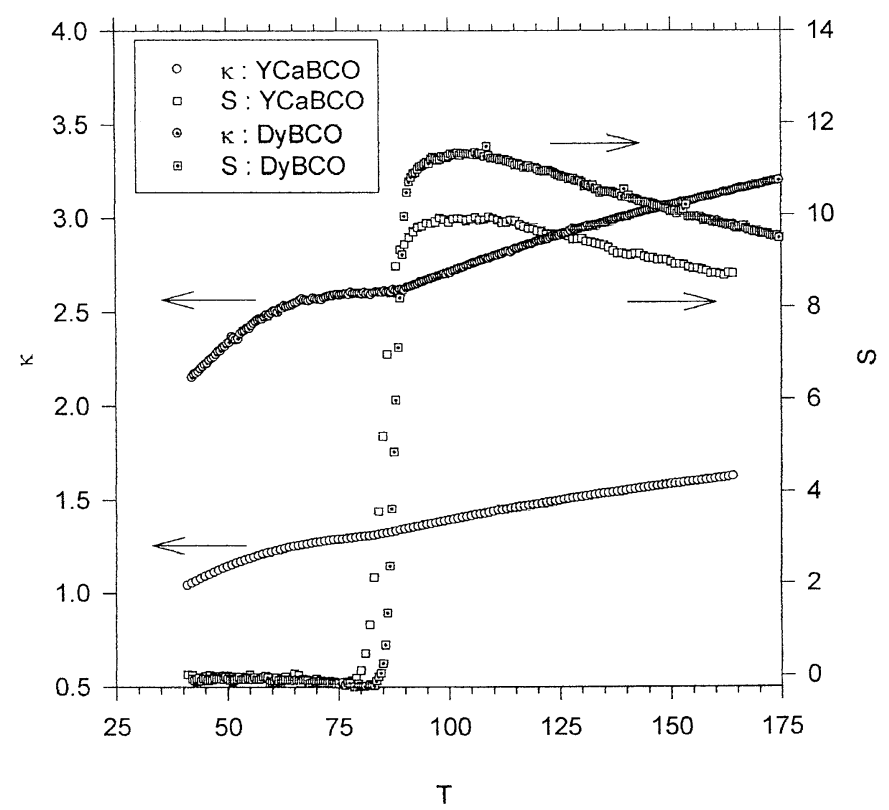

Fig. 4. Comparison between the thermal conductivity and the thermopower as function of temperature for $\mathrm{Y}_{1-x} \mathrm{Ca}_{x} \mathrm{Ba}_{2} \mathrm{Cu}_{3} \mathrm{O}_{7-\delta} \quad(x=0.1)$ and $\mathrm{Dy}_{1} \mathrm{Ba}_{2} \mathrm{Cu}_{3} \mathrm{O}_{7-\delta}$ polycrystals.

ceramics superconductors $\mathrm{Y}_{1-x} \mathrm{Ca}_{x} \mathrm{Ba}_{2} \mathrm{Cu}_{3} \mathrm{O}_{7-\delta} \quad(x=$ 0.1 ) and $\mathrm{Dy}_{1} \mathrm{Ba}_{2} \mathrm{Cu}_{3} \mathrm{O}_{7-\delta}$ in Figure 4. The thermal diffusivity $\alpha$ is plotted versus temperature for the $\mathrm{Y}_{1-x} \mathrm{Ca}_{x} \mathrm{Ba}_{2} \mathrm{Cu}_{3} \mathrm{O}_{7-\delta}(x=0.1)$ and $\mathrm{Dy}_{1} \mathrm{Ba}_{2} \mathrm{Cu}_{3} \mathrm{O}_{7-\delta}$ samples respectively in Figure 5. An inset show the same data on a log-log plot. For these samples, the specific heat as a function of temperature can be deduced (Fig. 6). Notice that the data are comparable to those found in the literature respectively for the thermal conductivity $[14,15]$, the thermopower $[16,17]$, the thermal diffusivity [18], and the specific heat [19], but obviously those data were obtained under different conditions and nonsimultaneous runs. 


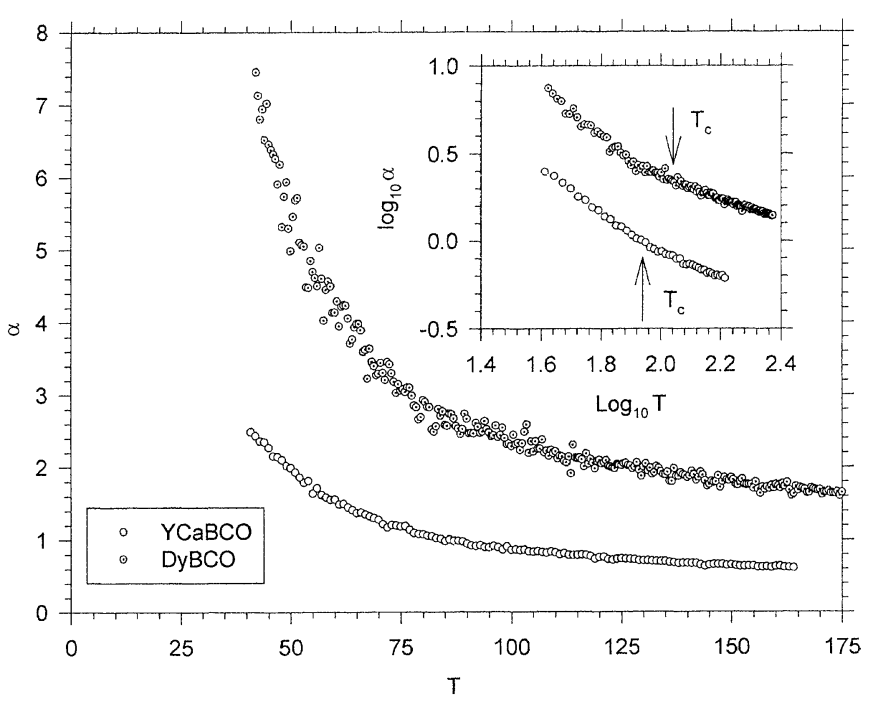

Fig. 5. Comparison between the thermal diffusivity for $\mathrm{Y}_{1-x} \mathrm{Ca}_{x} \mathrm{Ba}_{2} \mathrm{Cu}_{3} \mathrm{O}_{7-\delta}(x=0.1)$ and $\mathrm{Dy}_{1} \mathrm{Ba}_{2} \mathrm{Cu}_{3} \mathrm{O}_{7-\delta}$ polycrystals as a function of the temperature. Inset: comparison between the logarithm of the thermal diffusivity as a function of the logarithm of the temperature. The superconducting temperatures determined from electrical resistivity data are indicated by arrows.

The electrical resistivity $\rho(T)$ is shown respectively for the same $99.9 \%$ pure Goodfellow $\mathrm{Cu}$, $\mathrm{Y}_{1-x} \mathrm{Ca}_{x} \mathrm{Ba}_{2} \mathrm{Cu}_{3} \mathrm{O}_{7-\delta}(x=0.1)$ and $\mathrm{Dy}_{1} \mathrm{Ba}_{2} \mathrm{Cu}_{3} \mathrm{O}_{7-\delta}$ polycrystalline samples between $120 \mathrm{~K}$ and $40 \mathrm{~K}$ for further discussion. The electrical resistivity was measured in the same set-up (Fig. 1) but not simultaneously with the other properties. The electrical resistivity has been measured using the conventional four-probe method with the sample glued through the varnish GE7031 and cigarette paper directly to the sink. The voltage drop $\Delta V$ across the sample is measured with great accuracy. A one second pulse dc current $I$ is injected successively on both sides of the sample in order to avoid Joule and Peltier effect respectively. The electrical resistivity is given by $\rho=(V / I)(A / L)$, where $L$ is the distance between the voltage leads on the sample and $A$ is the cross section area of the sample as before. Notice the great precision of the data and their very smooth evolution without noise.

\section{Discussion}

Taking into account the convection and conduction thermal losses (high vacuum, long and thin themocouples respectively), we can first pin point the importance of the thermal losses by radiation and show how the radiation shield improves the data quality.

\subsection{Cu sample}

For the $\mathrm{Cu}$ sample the radiation losses induce data differences (Fig. 2) which can be estimated to be $15 \%$ and $45 \%$ for the thermal diffusivity and thermal conductivity respectively at room temperature. One can see that the thermal radiation losses induce a shift in amplitude of

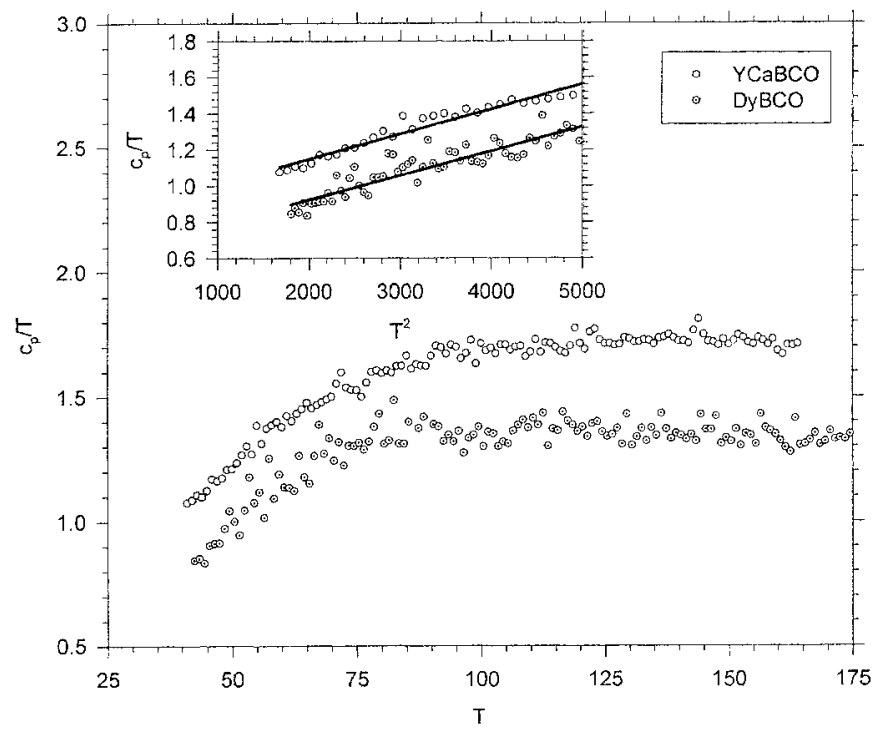

Fig. 6. Specific heat $\left(c_{\mathrm{p}} / T\right)$ as a function of temperature for $\mathrm{Y}_{1-x} \mathrm{Ca}_{x} \mathrm{Ba}_{2} \mathrm{Cu}_{3} \mathrm{O}_{7-\delta}(x=0.1)$ and $\mathrm{Dy}_{1} \mathrm{Ba}_{2} \mathrm{Cu}_{3} \mathrm{O}_{7-\delta}$ polycrystals as determined from data in Figures 4 and 5. Inset: comparison of $c_{\mathrm{p}} / T$ data as a function of $T^{2}$ for these samples.

the data but no change in qualitative behavior. The shift of the data without the radiation is due to overestimating the heat flux through the sample due mainly to a "neglect of the radiation losses", since the cover is kept at the sink temperature, i.e. 2 to $3 \mathrm{~K}$ lower than the temperature of the sample. The specific heat data compared for both experimental situations is seen in Figure 3. The neglect of radiation losses (the absence of radiation shield) is clearly seen inducing a $50 \%$ error at room temperature. In the presence of radiation shields one can see that the thermal diffusivity, the thermal conductivity, the thermopower and the electrical resistivity (Fig. 7) data of $\mathrm{Cu}$ are comparable to those found in the literature $[20-24]$ when measured independently. At room temperature the difference between our best data and the most reliable literature data is about $6 \%$ for the thermal diffusivity [20], and $8 \%$ for the thermal conductivity [21,22], $5 \%$ for the thermopower [23], and $2 \%$ for the electrical resistivity (Fig. 7) [24]. This confirms the error bar estimated in [1] and in Section 2.3. Notice that some difference between our results and those in the literature can be easily tied to a different (though small) impurity concentration. In our sample the RRR of the electrical resistivity is about 100 . If the sample purity content is thus a little bit larger than for other best standard samples, this easily leads to explain the intrinsically slightly smaller value of the thermal diffusivity and thermal conductivity found here above.

In Figure 3 we also present the low temperature $(T<$ $55 \mathrm{~K})$ specific heat $c_{\mathrm{p}}$ of this $99.9 \%$ pure Goodfellow $\mathrm{Cu}$ sample as function of temperature $T$ (on a $c_{\mathrm{p}} / T$ vs. $T^{2}$ plot) in presence or absence of radiation shield as deduced from the rough data on the thermal conductivity and the thermal diffusivity. The linear fit $\left(c_{\mathrm{p}} / T=\gamma+\lambda T^{2}\right)$ of 


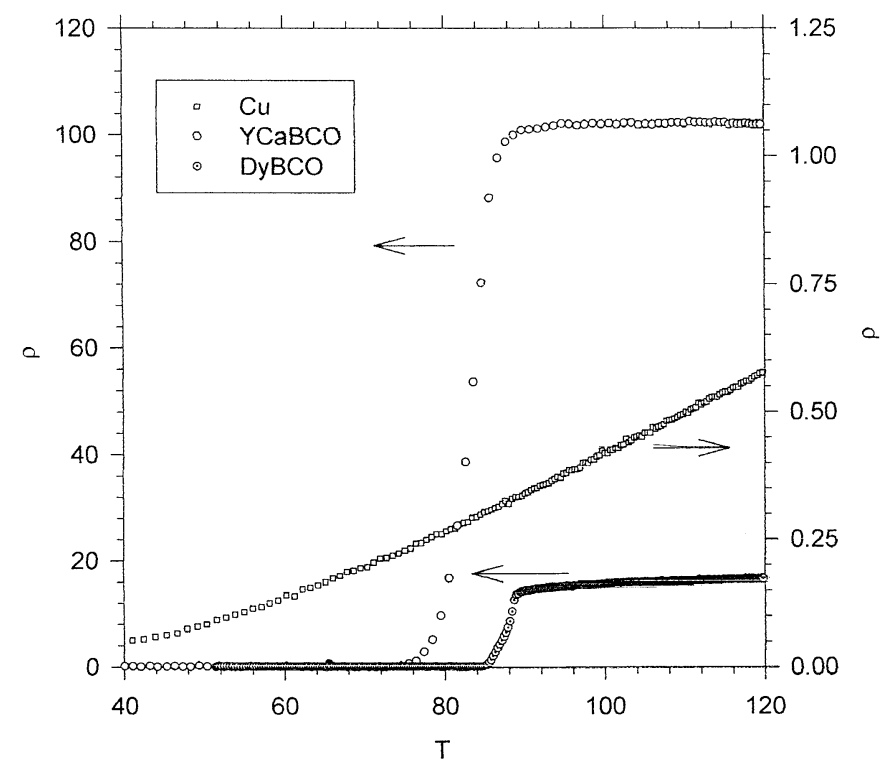

Fig. 7. Electrical resistivity $\rho(T)$ as function of temperature of a $99.9 \%$ pure $\mathrm{Cu}$ sample, $\mathrm{Y}_{1-x} \mathrm{Ca}_{x} \mathrm{Ba}_{2} \mathrm{Cu}_{3} \mathrm{O}_{7-\delta}(x=0.1)$, and $\mathrm{Dy}_{1} \mathrm{Ba}_{2} \mathrm{Cu}_{3} \mathrm{O}_{7-\delta}$ polycrystals.

the data leads to the determination of the so-called initial Debye temperature $\theta_{\mathrm{D}}$. The slopes $\lambda$ of the linear fit are $7.1 \times 10^{-5} \mathrm{~J} / \mathrm{mol} \mathrm{K}^{4}$ and $1.16 \times 10^{-4} \mathrm{~J} / \mathrm{mol} \mathrm{K}^{4}$ for the case or with and without a radiation shield presence respectively. The Debye temperatures are estimated to be $302 \mathrm{~K}$ and $256 \mathrm{~K}$ respectively from $\theta_{\mathrm{D}}^{3}=234 R \lambda^{-1}$ where $R$ is the ideal gas constant. The former is in good agreement with the value $\theta_{\mathrm{D}}=309 \mathrm{~K}$ found in the literature [25].

Notice that to our knowledge there is no theory of the thermal diffusivity of metals except through the ratio of the thermal conductivity and the specific heat. Moreover it is also known that the thermal conductivity decay at high temperature ( $T>70 \mathrm{~K}$ here) is also not well reproduced by standard formulae even those of Berman [26] or Ziman [27]. However the Wiedmann-Franz law can be used to predict the electronic contribution at high temperature.

\subsection{Ceramics samples}

The data on ceramics samples (Figs. 4-6) is sufficiently precise that one can easly take a numerical derivative. This is useful because the superconductivity critical (Landau-Ginzburg) temperature $T_{\mathrm{c}}$ is best determined from the maximum derivative of the electrical resistivity. We find $T_{\mathrm{c}}$ to be $85 \mathrm{~K}$ and $89 \mathrm{~K}$ respectively for such $\mathrm{Y}_{1-x} \mathrm{Ca}_{x} \mathrm{Ba}_{2} \mathrm{Cu}_{3} \mathrm{O}_{7-\delta}(x=0.1)$ and $\mathrm{Dy}_{1} \mathrm{Ba}_{2} \mathrm{Cu}_{3} \mathrm{O}_{7-\delta}$ systems (Fig. 7). From the thermopower data, the inflexion point corresponding to the Landau-Ginzburg temperature is also found to be at 85 and $89 \mathrm{~K}$ respectively. Below the critical temperature the value of the thermal conductivity is remarkably larger and higher for the $\mathrm{Dy}_{1} \mathrm{Ba}_{2} \mathrm{Cu}_{3} \mathrm{O}_{7-\delta}$ than for the $\mathrm{Y}_{1-x} \mathrm{Ca}_{x} \mathrm{Ba}_{2} \mathrm{Cu}_{3} \mathrm{O}_{7-\delta}$ $(x=0.1)$ sample. Notice that a hump even though small is nevertheless seen on the $\mathrm{Dy}_{1} \mathrm{Ba}_{2} \mathrm{Cu}_{3} \mathrm{O}_{7-\delta}$ sample. This shows the high quality of the method.
Usually the thermal conductivity of insulators or metallic systems decreases or is a constant at high temperature [27]. The increase seen for the thermal conductivity of the ceramics cuprates at high temperature (Fig. 4) is a well-known puzzling but standard feature. Such an increase is also found in amorphous systems like glasses [28].

In both cases, i.e. for $\mathrm{Y}_{1-x} \mathrm{Ca}_{x} \mathrm{Ba}_{2} \mathrm{Cu}_{3} \mathrm{O}_{7-\delta}(x=$ 0.1 ) and $\mathrm{Dy}_{1} \mathrm{Ba}_{2} \mathrm{Cu}_{3} \mathrm{O}_{7-\delta}$, the thermal diffusivity shown in Figure 5 shows a break in slope between different power laws characterizing the normal state and the superconducting state. The cross-over temperature is found to be equal to $85 \pm 10 \mathrm{~K}$ and $89 \pm 10 \mathrm{~K}$ in the $\mathrm{Y}_{1-x} \mathrm{Ca}_{x} \mathrm{Ba}_{2} \mathrm{Cu}_{3} \mathrm{O}_{7-\delta}(x=0.1)$ and in $\mathrm{Dy}_{1} \mathrm{Ba}_{2} \mathrm{Cu}_{3} \mathrm{O}_{7-\delta}$, and is very close to each $T_{\mathrm{c}}$ (Fig. 7 ) as determined from the other transport coefficients. This cross-over may originate from different electronic contributions [30,31], on both sides of $T_{\mathrm{c}}$ since the thermal diffusivity like the thermal conductivity is proportional to the mean free path of the heat carriers. Notice that the superconducting mechanism is likely not very different in both samples. Moreover, the thermal diffusivity is usually considered to be independent of the number of carriers. We can conclude from the above that impurities and defects scattering contribution are greater in the $\mathrm{Y}_{1-x} \mathrm{Ca}_{x} \mathrm{Ba}_{2} \mathrm{Cu}_{3} \mathrm{O}_{7-\delta}(x=0.1)$ compound than in the $\mathrm{Dy}_{1} \mathrm{Ba}_{2} \mathrm{Cu}_{3} \mathrm{O}_{7-\delta}$ one. This is in agreement with the absolute values of the electrical resistivity (Fig. 7), the thermoelectric power and the thermal conductivity measurements (Fig. 4).

The specific heat $c_{\mathrm{p}}$ can be calculated from the expression $\alpha=\kappa / d c_{\mathrm{p}}$ where the density $d$ is measured using the formula $d=m / V$ where $m$ is the mass and $V$ the volume of the samples (the density temperature variation is neglected since in the temperature range of interest the correction from the volume expansion coefficient is of the order of $10^{-3}$ ). Even though the specific heat is mainly due to phonons, a peak in the specific heat is well observed in the $\mathrm{Dy}_{1} \mathrm{Ba}_{2} \mathrm{Cu}_{3} \mathrm{O}_{7-\delta}$ compound close to $85 \mathrm{~K}$ (Fig. 6), corresponding to a 7\% electronic extra contribution of superconducting origin at $T_{\mathrm{c}}$. The peak at $T_{\mathrm{c}}$ is not so apparent in the case of the $\mathrm{Y}_{1-x} \mathrm{Ca}_{x} \mathrm{Ba}_{2} \mathrm{Cu}_{3} \mathrm{O}_{7-d}$ $(x=0.1)$ sample which is a "bad sample" since the number of weak links and porosity are rather large as seen from the electrical resistivity amplitude (Fig. 7). Notice the relative values of $\kappa(T), \alpha(T), S(T)$ and $\rho(T)$ for both ceramics. The consistency of relative values indicates that the sample have a markedly different porosity.

The slopes of the linear fit of Figure 6 for the specific heat of $\mathrm{Y}_{1-x} \mathrm{Ca}_{x} \mathrm{Ba}_{2} \mathrm{Cu}_{3} \mathrm{O}_{7-\delta} \quad(x=0.1)$ and $\mathrm{Dy}_{1} \mathrm{Ba}_{2} \mathrm{Cu}_{3} \mathrm{O}_{7-\delta}$ are $1.4 \times 10^{-4} \mathrm{~J} / \mathrm{mol} \mathrm{K}^{4}$ and $1.3 \times 10^{-4} \mathrm{~J} / \mathrm{mol} \mathrm{K}^{4}$ respectively. The Debye temperatures are estimated to be $242 \mathrm{~K}$ and $244 \mathrm{~K}$. A wide range of values $\theta_{\mathrm{D}}$, i.e. between 320 and $380 \mathrm{~K}$, can be found in the literature [19] even for $\mathrm{Y}_{1} \mathrm{Ba}_{2} \mathrm{Cu}_{3} \mathrm{O}_{7-\delta}$. The values found here are merely indicative of course since the temperature range is not fully appropriate for that sort of linear fit. Moreover the Debye temperature much depends on the oxygen and metallic ion content. Therefore various data cannot meaningfully be compared here to each other without much further discussion outside the scope of this paper. 


\section{Conclusions}

In the previous sections we have presented a set-up and method in order to measure transport properties in a thermal gradient simultaneously. We have illustrated the method and briefly shown a few cases. In particular we obtain reliable data for the thermal diffusivity at rather low temperature, - measurements which are not available in the literature to our knowledge except in an indirect way. See, e.g. the case of $\mathrm{Cu}$ below $100 \mathrm{~K}$ here above.

With respect to the methods and set-ups found in the literature $[32,33]$ our technique has two main advantages. The technique allows one to analyse four thermal properties, $\kappa(T), S(T), \alpha(T)$ and $c_{\mathrm{p}}(T)$ simultaneously at the same temperature for solid samples using three absolute thermocouples. The case of simultaneously measuring the thermal diffusivity together with the other properties is new. The technique is rather fast as well.

One technical reason for the positive aspect of the above can be traced to the fact that the temperature is measured with great accuracy as a function of time. Indeed after stabilization of the sample holder temperature, the sample temperature rise is measured before, during and after the pulse successively by each thermocouple. The interpolation of the measured temperature can be done on a small time interval and the calculated temperature can be determined at small space intervals. In this report we have taken $\Delta t$ and $\Delta x$ to be $0.25 \mathrm{~s}$ and $0.25 \mathrm{~mm}$ respectively. Those conditions are of course obtained because of the sensitive, precise and fast set of apparatuses which are available on the market in order to measure and control the temperature systems. Such systems allow us also to be finely acquiring the experimental data and treating it thereafter in a fine and efficient way.

The error bars have been shown to be sufficiently small. Moreover it has been shown that many data points can be taken. Data treatments are thus more feasible and useful to test theoretical models or calculations

Part of this work has been financially supported by Action de Recherche Concertée (ARC) 94/99-174, Communauté Française de Belgique Direction de la Recherche Scientifique. S. Dorbolo acknowledges financial support from FRIA. We thank Professor H.W. Vanderschueren for allowing us to use the M.I.E.L equipment and for his constant interest. Part of this work was financially supported through a Belgium-Poland Scientific agreement. J.M. and M.A. are grateful to KBN (Warsaw) for supporting this agreement. I. Nedkov acknowledges financial support from the National Fund of Scientific Research, Bulgaria, under Grant No TH-539 and CGRI grants.

\section{References}

1. H. Bougrine, Ph.D. thesis University of Liege, Belgium, 1994.

2. H. Bougrine, M. Pekala, Supercond. Sci. Technol. 10, 1 (1997).

3. W.L. Kennedy, P.H. Sidles, G.C. Danielson, Adv. Energy Conv. 2, 53 (1962).
4. J.H. McTaggart, G.A. Slack, Cryogenics 9, 384 (1996).

5. M.J. Laubitz, Compendium of Thermophysical Property Measurements Methods, edited by K.D. Maglic (Plenum, New York, 1984) Vol. 1.

6. Z. Henkie, P.J. Markowski, A. Wojakowski, Ch. Laurent, J. Phys. E 20, 40 (1987).

7. J.L. Tallon, C. Bernhard, H. Shaked, R.L. Hitterman, J.D. Jorgensen, Phys. Rev. B 51, 12911 (1995).

8. I. Nedkov, Supercond. Sci. Technol. 10, 18 (1997).

9. I. Nedkov, S. Miteva, T. Koutsarova, in Abstracts Proc., 3rd European Conference on Applied Superconductivity, EUCAS'97 (13Ge/69, Netherlands, 1997), p. 187.

10. J. Madsen, J. Trefny, J. Phys. E 21, 636 (1988).

11. C.S. McMenamin, D.F. Brewer, T.E. Hargreaves, N.E. Hussey, C. Moreno, A.L. Thomson, J.P. Bird, Physica B 191-196, 21 (1994).

12. J. Crank, The Mathematics of Diffusion, 2nd ed. (Clarendon Press, London, 1973), p. 144.

13. Y.S. Touloukian, E.H. Buyco, Thermophysical Properties of Matter: Specific heat (IFI Plenum, New York, 1970), Vol. 4.

14. M. Houssa, M. Ausloos, R. Cloots, H. Bougrine, Phys. Rev. B 56, 802 (1997).

15. M. Ikebe, K. Fujishiro, K. Nakasato, K. Noto, Physica C 263, 309 (1996).

16. M. Ausloos, Ch. Laurent, Phys. Rev. B 37, 611 (1988).

17. M. Pekala, H. Bougrine, J. Azoulay, M. Ausloos, Supercond. Sci. Technol. 8, 660 (1995).

18. V. Calzona, M.R. Cimberli, C. Ferdeghini, M. Putti, C. Rizzuto, A.S. Siri, Europhys. Lett. 13, 181 (1990).

19. A. Junod, D. Eckert, G. Triscone, J. Phys. Cond. Matter 1, 8021 (1989).

20. Y.S. Touloukian, R.W. Powell, C.Y. Ho, M.C. Nicolau, Thermophysical Properties of Matter: Thermal Diffusivity (IFI Plenum, New York, 1973), vol. 10.

21. Y.S. Touloukian, R.W. Powell, C.Y. Ho, P.G. Klemens, Thermophysical Properties of Matter: Thermal Conductivity (IFI Plenum, New York, 1970), vol. 1.

22. G.K. White, M.L. Minges, Int. J. Thermophys. 18, 1269 (1997).

23. D.K.C. Mac Donald, Principles of Thermoelectricity (Wiley, New York, 1962).

24. G.K. White, Experimental Techniques in Low Temperature Physics, 3rd ed. (Clarendon Press, Oxford, 1979).

25. M.N. Khlopkin, in Handbook of Physical Quantities, edited by I.S. Grigoriev, E.Z. Meilikhov (CRC Press, Boca Raton, 1997), p. 264.

26. R. Berman, Thermal Conduction in Solids (Clarendon Press, Oxford, 1976).

27. J.M. Ziman, Electrons and Phonons (Clarendon Press, Oxford, 1960).

28. V.G. Karpov, D.A. Parshin, Sov. Phys. JETP 61, 1308 (1985) [Zh. Eksp. Teor. Fiz. 88, 2212 (1985).

29. E.B. Gel'man, in Handbook of Physical Quantities, edited by I.S. Grigoriev, E.Z. Meilikhov (CRC Press, Boca Raton, 1997) p. 283.

30. M. Houssa, M Ausloos, Physica C. 257, 321 (1996).

31. M. Houssa, Ph.D. thesis University of Liège, Belgium, 1996.

32. M. Ikebe, H. Fujishiro, T. Naito, K. Noto, J. Phys. Soc. Jpn. 63, 3107 (1994).

33. C. Meis, A.K. Froment, D. Moulinier, J. Phys. D 26, 520 (1993). 